\title{
Performance Metrics for Self-Positioning Autonomous MANET Nodes
}

\author{
Janusz Kusyk, ${ }^{1}$ Jianmin Zou ${ }^{2}$, Stephen Gundry ${ }^{2}$, Cem Safak Sahin ${ }^{3}$ \\ and M. Ümit Uyar ${ }^{2}$ \\ ${ }^{1}$ The United States Patent and Trademark Office, Alexandria, VA, USA; \\ e-mail: janusz.kusyk@uspto.gov \\ ${ }^{2}$ The Department of Electrical Engineering, The City College of New York, NY, \\ USA; e-mail: \{jzou00, sgundry00\}@citymail.cuny.edu, uyar@ccny.cuny.edu \\ ${ }^{3}$ BAE Systems - AIT, Burlington, MA, USA; e-mail: csafaksahin@gmail.com
}

Received 20 May 2013; Accepted 15 July 2013

\begin{abstract}
We present quantitative techniques to assess the performance of mobile ad hoc network (MANET) nodes with respect to uniform distribution, the total terrain covered by the communication areas of all nodes, and distance traveled by each node before a desired network topology is reached. Our uniformity metrics exploit information from a Voronoi tessellation generated by nodes in a deployment territory. Since movement is one of the most power consuming tasks that mobile nodes execute, the average distance traveled by each node (ADT) before the network reaches its final distribution provides an important performance assessment tool for power-aware MANETs. Another performance metric, network area coverage (NAC) achieved by all nodes, can demonstrate how efficient the MANET nodes are in maximizing the area of operation. Using these metrics, we evaluate our node-spreading bio-inspired game (BioGame), that combines our force-based genetic algorithm (FGA) and game theory to guide autonomous mobile nodes in making movement decisions. Our simulation experiments demonstrate that these performance evaluation metrics are good indicators for assessing the efficiency of node distribution methods.
\end{abstract}

Journal of Cyber Security and Mobility, Vol. 2, 151-173.

doi 10.13052/jcsm2245-1439.223

(C) 2013 River Publishers. All rights reserved. 


\section{J. Kusyk et al.}

Keywords: Topology control, MANETs, node-spreading, uniformity measures, Voronoi tessellation, area coverage, game theory, bio-inspired algorithms.

\section{Introduction}

Mobile ad hoc networks (MANETs) are useful for many commercial and military applications where network coverage is needed over a terrain without an established infrastructure. Autonomous topology control algorithms aim to provide a method to deploy mobile assets without a centralized controller such that MANETs are scalable and robust to node failures. In this context, it is advantageous when the MANET topology has reduced sensing overshadows, limited blind spots, enhanced spectrum utilization, and simplified routing procedures while lowering power consumed by each node. Achieving these objectives necessitate that autonomous nodes in MANETs (a) place themselves over an unknown geographical terrain in order to obtain a uniform network distribution, (b) reduce the total distance traveled before overall network objectives are reached, and (c) preserve network connectivity while attaining positions that ensure a high coverage of the area by all nodes. In this article, we present quantitative methods to evaluate performance of node selfpositioning techniques with respect to uniformity of distribution of nodes, average distance traveled (ADT) by each autonomous vehicle, and the total area coverage (NAC) obtained by all nodes.

The uniform distribution of mobile nodes is often a desired network topology that helps to prolong network lifespan by ensuring that nodes deplete their energy resources evenly. When MANET nodes are uniformly distributed, they are able to equally share sensing and communication tasks, hence the likelihood that a single node ceases to function much earlier than expected due to power exhaustion is reduced. In order to gauge the performance of a MANET with respect to its uniform distribution, we define metrics based on Voronoi regions generated by the nodes.

Since a node's movement is typically the most energy-consuming operation performed by autonomous vehicles, reducing the distance that nodes travel is an important objective. ADT provides a realistic metric for evaluating self-spreading algorithms where preserving scarce energy resources is imperative.

Gathering information about operational environments to provide mission-critical data is often the main motivation for deployment of MANETs. In order to adequately utilize the communication coverage of deployed nodes, 
network topologies that increase NAC while preserving connectivity are often needed. Consequently, NAC provides an intuitive metric to assess how well existing communication coverage resources are utilized.

Our metrics offer design-aiding techniques for power-aware MANETs where balancing desired network performance with power-limiting constraints are imperative to maximize the utilization of deployable resources. We demonstrate the practicality of Voronoi-based uniformity, ADT, and NAC metrics by applying them to our node-spreading bio-inspired game (BioGame). BioGame combines our force-based genetic algorithm (FGA) and game theory (GT) concepts to guide autonomous mobile nodes in selecting locations that improve uniformity, and network coverage while limiting the distance that nodes travel. In this paper, we present a formal definition of our BioGame and compare its simulation results to the outcomes attained by mobile nodes guided by our FGA alone.

The rest of this article is organized as follows. A brief overview of the related research is presented in Section 2. Section 3 formally introduces our uniformity metrics as well as ADT and NAC evaluation techniques. In Sections 4 and 5, we define our BioGame and analyze its performance by conducting simulation experiments, respectively.

\section{Related Work}

In this section, we present background to Voronoi tessellation and topology control methods as well as our earlier research. An interested reader can find an extensive analysis of GT in the work by Fudenberg and Tirole [8]. Holland [13] and Mitchell [21] present the essentials of genetic algorithms (GAs).

\subsection{Background}

Voronoi tessellation has been applied to analyze biological cell models and the territorial behavior exhibited by animals $[3,10]$. Lu et al. [19], use centroidal Voronoi tessellation for the efficient placement of vertices when rendering surfaces in computer graphics. Voronoi diagrams can also be applied to facial recognition algorithms, as demonstrated by Abbas et al. [1]. Bash and Desnoyers [2] present a distributed method for nodes in a sensor network that use Voronoi region boundaries to assist in achieving improved load balancing and energy conservation. Other Voronoi-based applications include quality measures for point distribution in an area, as presented by 
Nguyen et al. [22], and optimal distribution of resources through applications of a centroidal Voronoi tessellation method, examined by Du et al. in [7].

Topology control of mobile nodes in MANETs has been studied in various contexts. In [29] and [4], nodes with a fixed configuration in a MANET dynamically adjust their power levels to achieve $k$-connectivity. Garro et al. [9] present a bio-inspired algorithm that allows mobile nodes to find unobstructed paths to predefined targets. Differential evolution (DE) has been successfully applied to decentralized robotic applications. In [28], Vahdat et al. present DE and particle swarm optimization that are applied to the global localization of mobile robots. In [26], DE is used for MANETs to improve the performance of routing protocols. DE is used as the mechanism for MANET nodes to choose cluster heads, as shown in [5]. Managing the movement of nodes in network models where each node is capable of changing its own spatial location has been approached by employing concepts of potential fields [14], a Lloydbased algorithm [6], and various GA-based decentralized topology control mechanisms [24].

\subsection{Our Earlier Research}

In our earlier work [11,17], we presented three distinct methods for autonomous MANET nodes to position themselves over unknown deployment areas using various GT, evolutionary GT, and FGA concepts. We introduced a forcebased genetic algorithm topology control approach for uniform deployment of autonomous vehicles over a two-dimensional unknown area in [25, 27]. In [12], we introduced a differential evolution-based topology control mechanism, called TCM-DE, which we modeled as an inhomogeneous Markov chain to demonstrate its convergence towards an adequately separated final distribution of mobile nodes. We studied models that combine various GT and genetic algorithms concepts for autonomous MANET nodes positioning themselves over an unknown deployment areas in $[15,16,30]$.

\subsection{Contribution of This Paper}

The initial concepts used to evaluate the performance metrics of selfpositioning autonomous MANET nodes were introduced in [11,18]. In this paper, we introduce the formal definition of our BioGame. Then, using our performance metrics, we formally analyze the performance of our BioGame and FGA with respect to the average distance traveled, uniform distribution of nodes, and area coverage. We verify formal analysis results with simu- 
lation experiments and show that both BioGame and FGA can be effective in providing uniform node distribution. However, BioGame, which utilizes game theory, can make better informed decisions and at the same time reduce traveling distance for nodes. This paper presents a formal definition of our BioGame together with its convergence properties. Since the nodes running BioGame base their decisions not only on the expected improvement of their own locations but also on the possible movements of their near neighbors, we are able to demonstrate that BioGame provides better informed movement decisions for the mobile nodes. Using simulation experiments, we verify that the performance of MANET nodes guided by BioGame is better than by our FGA alone.

\section{Performance Evaluation Methods for MANETs}

In this section, we present quantitative methods for assessing performance of MANETS with respect to the uniform distribution of mobile nodes, NAC, and ADT.

\subsection{Uniformity Metrics}

Equally distanced and connected mobile nodes are necessary to achieve many network goals. A uniform distribution of mobile nodes helps to simplify high-level network communication and routing operations as well as provide adequate area coverage for environment-sensing purposes. Furthermore, since the lifespan of a MANET under limited-power conditions often depends on the continuous operation of all nodes, it is important to ensure that the nodes deplete their energy resources evenly and to limit the number of nodes that cease to function prematurely. In uniformly distributed networks, where each node has the same sensing area and distance to its neighbors, power utilized by every mobile node to perform its tasks is similar and, consequently, prolonged uninterrupted operation of a MANET can be accomplished.

To gauge the performance of MANETs with respect to the uniformity of autonomous node distribution, we introduce metrics based on various quantities associated with the Voronoi tessellation [23] of the deployment terrain. Our Voronoi tessellation metric associates each node $n_{i}$ with a Voronoi region $V_{i}$ such that all locations that are closer to $n_{i}$ than to any of the other nodes 


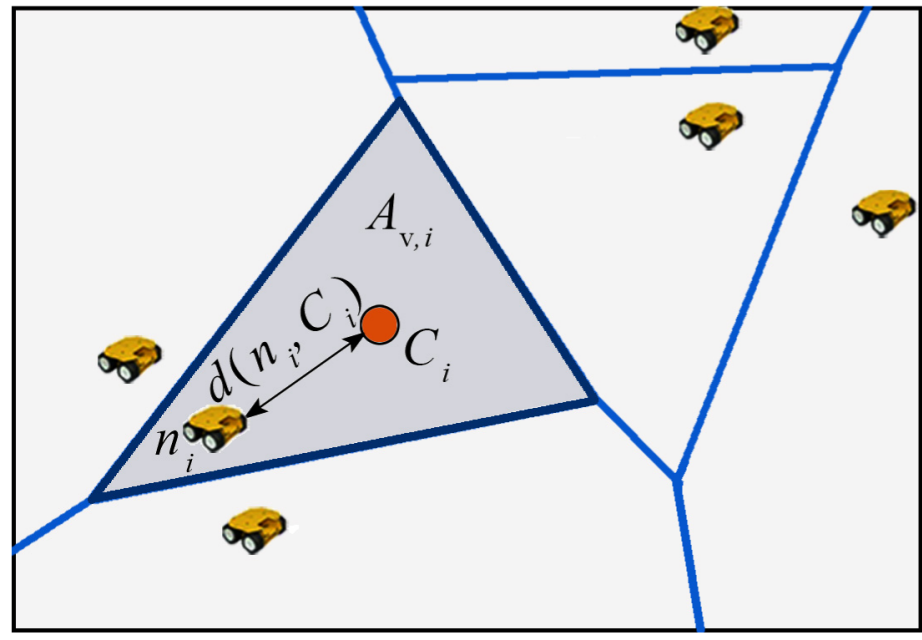

Figure 1 The Voronoi tessellation of a rectangular area.

are parts of its $V_{i}$. The Voronoi region for each MANET node is defined as

$$
V_{i}=\left\{\omega \in \Omega: d\left(n_{i}, \omega\right)<d\left(n_{j}, \omega\right), \forall_{n_{j} \in I \backslash\left\{n_{i}\right\}}\right\}
$$

where $\Omega$ represents the set of all positions in the deployment area, $I$ is a set of all players (nodes), and $d\left(n_{i}, \omega\right)$ represents the Euclidean distance between node $n_{i}$ and a location in the deployment area (i.e., $\left(x_{i}, y_{i}\right)$ and $\left.\left(x_{\omega}, y_{\omega}\right)\right)$. The Voronoi tessellation of a deployment terrain is a collection of all nodes' Voronoi regions. Let the area of $V_{i}$ be defined as $A_{\mathrm{v}, i}$ and $C_{i}$ be the center of mass of region $V_{i}$. Figure 1 presents a tessellation of the rectangular constant terrain depicting basic quantities associated with each Voronoi region.

In Figure 1, the darker region represents the area, $A_{\mathrm{v}, i}$ of node $n_{i}$ 's Voronoi cell $V_{i}$. The parameter $d\left(n_{i}, C_{i}\right)$ denotes the distance to the center of mass of generated by node $n_{i}$ 's Voronoi region. For clarity of presentation, Figure 1 does not depict any values associated with the other five nodes.

We introduce two methods for measuring the uniform distribution of MANET nodes over a deployment terrain. The first metric, called $\mathcal{U}_{A}$, exploits differences in sizes of the areas for Voronoi regions generated by the nodes. We define $\mathcal{U}_{A}$ as

$$
\mathcal{U}_{A}=\frac{1}{\bar{A}_{\mathrm{v}}} \sqrt{\frac{1}{|I|} \sum_{n_{i} \in I}\left(A_{\mathrm{v}, i}-\bar{A}_{\mathrm{v}}\right)^{2}}
$$



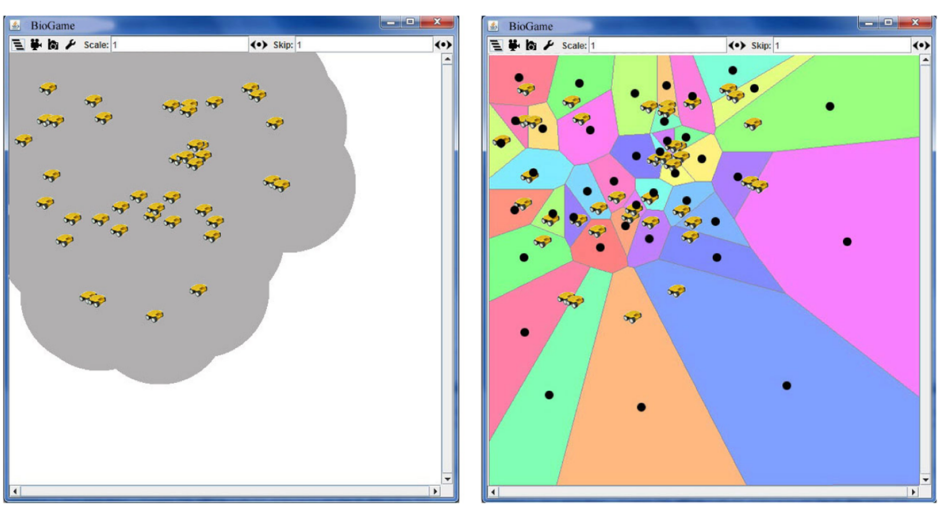

(a)
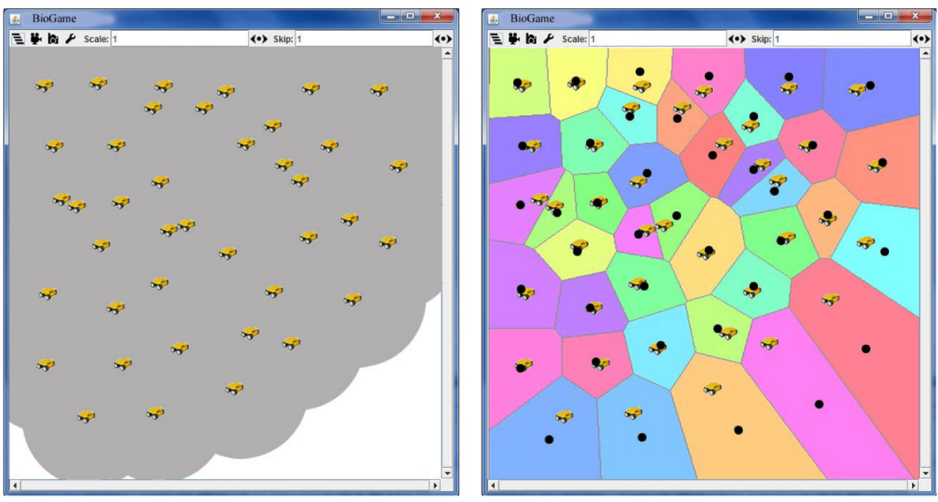

(b)
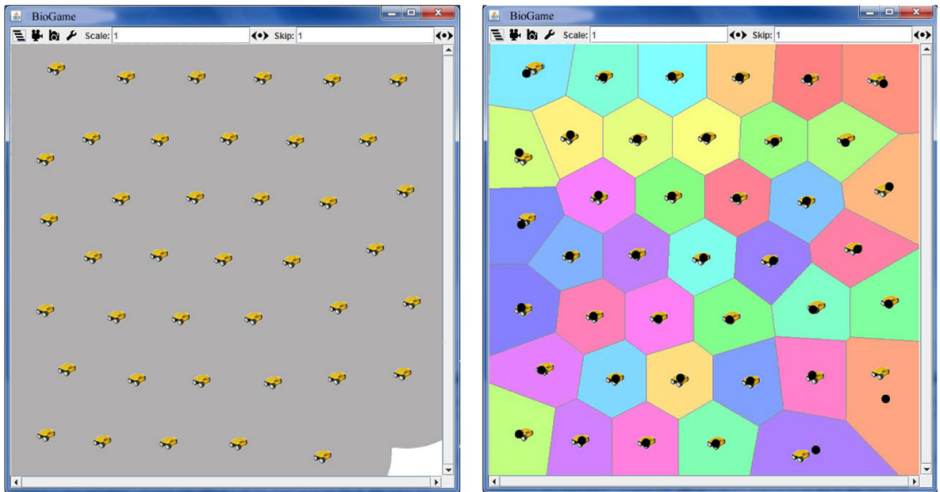

(c)

Figure 2 Examples of node distributions and corresponding Voronoi tessellations obtained by BioGame at step: (a) $t=5$; (b) $t=15$; and (c) $t=50$. The center of mass for each Voronoi region is marked by a black dot. 
where $\bar{A}_{\mathrm{v}}$ is the arithmetic mean of $A_{\mathrm{v}, i}$ for all $n_{i} \in I$ and $|I|$ denotes a total number of nodes in the network.

If mobile nodes are equally separated, the size of the Voronoi cell area for each node located in the interior of the deployment terrain is equal. Slight variations in Voronoi regions exist near the boundaries of the deployment territory. Therefore, the tessellation of the deployment area that closely resembles collection of congruent regular hexagons reflects a desirable node distribution. The metric $\mathcal{U}_{A}$ approaches zero as autonomous mobile agents improve their locations towards a uniform network distribution, where nodes cannot improve their positions any further.

Figure 2 shows three sample node distributions achieved by our BioGame and the Voronoi tessellations associated with them. The center of mass for each Voronoi region is marked by a black dot. The values of $\mathcal{U}_{A}$ for the topologies depicted in Figures 2(a), (b), and (c) are 1.6, 0.6, and 0.2, respectively, which are consistent with improvement achieved by the network at these steps.

Our second metric for network uniformity is based on the distance between the location of a node $n_{i}$ and the center of mass of its Voronoi region $C_{i}$. In a given topology, the center of mass $C_{i}$ indicates the preferred location for node $n_{i}$ in order to best monitor its surrounding. The distance between the location of $n_{i}$ and $C_{i}$ indicates how close its present position is to the ideal position. The uniformity measure $\mathcal{U}_{C}$ is defined as

$$
\mathcal{U}_{C}=\frac{1}{|I|} \sum_{n_{i} \in I} d\left(n_{i}, C_{i}\right)
$$

where $d\left(n_{i}, C_{i}\right)$ is the Euclidean distance between the present position of $n_{i}$ and the center of its Voronoi region $C_{i}$ (Figure 1).

When a network converges to a uniform distribution, the separation among neighboring nodes equalizes and for all $n_{i} \in I$, and the distance $d\left(n_{i}, C_{i}\right)$ approaches zero. For example, the uniformity measures $\mathcal{U}_{C}$ for the topologies depicted in Figures 2(a), (b), and (c), are 7.8, 3.1, and 0.9, respectively. Consequently, in both of our metrics $\mathcal{U}_{A}$ and $\mathcal{U}_{C}$, the smaller value achieved by the network indicates the better placement of nodes.

\subsection{Average Distance Traveled}

Another important metric for assessing the performance of node selfspreading algorithms is ADT. Let $s_{i}^{t}$ represent a strategy of player $n_{i}$ at time $t$ that corresponds to the spatial coordinates of $n_{i}$ (i.e., $\left.s_{i}^{t}=\left(x_{i}^{t}, y_{i}^{t}\right)\right)$. Further- 
more, for all $t \geq 0$, let $d\left(n_{i}^{0}, n_{i}^{t}\right)$ denote the total distance traveled by $n_{i}$ up to time $t$. We define $\operatorname{ADT}(t)$ as the average distance traveled by a node until time $t$ as

$$
\operatorname{ADT}(t)=\frac{1}{|I|} \sum_{n_{i} \in I} d\left(n_{i}^{0}, n_{i}^{t}\right)
$$

In our simulation experiments, as $t$ increases, the value of ADT never decreases (i.e., ADT is a monotonically increasing function). The rate that ADT grows is an indicator of the dynamic nature of the network. As the network reaches a uniform distribution, where nodes are not able to improve their positions any further, the derivative of ADT is zero.

\subsection{Network Area Coverage}

NAC is defined as a ratio of the coverage achieved by the communication areas of all nodes to the total deployment terrain. If any part of the region is covered by more than one mobile node, the overlapped area is included in NAC calculations only once. Also, only the part of node's coverage area that falls within the area of deployment counts towards NAC. Let $A_{\mathrm{C}, i}$ denote the area covered by node $n_{i}$ and $A_{\mathrm{c}}$ be the size of the area of deployment. We formally define NAC as

$$
\mathrm{NAC}=\frac{\bigcup_{n_{i} \in I} A_{\mathrm{C}, i}}{A_{\mathrm{C}}}
$$

where $\bigcup$ represents the union of all coverage areas of subscribed nodes. A NAC value of one implies that the entire area is fully covered. Hence, obtaining the highest possible NAC by mobile agents is one of the goals for our game-theoretic and bio-inspired node spreading techniques.

\section{Our BioGame}

In our BioGame, each mobile node makes movement decisions based solely on local data. First, a node runs our novel FGA to find a set of preferred next locations and evaluates them through the spatial game set up among itself and its current neighbors. In BioGame, a set $I$ of $m$ players represents all active nodes in the network and for all $n_{i} \in I$, a set of strategies $S_{i}$ stands for the possible locations into which player $n_{i}$ can move. Let $N_{i}$ denote the set of neighbors of node $n_{i}$ in its communication range $R_{C}$, which defines $n_{i}$ 's sensing and communication areas. Strategy profile $s$ for player $n_{i}$ represents strategies of all nodes in $\left\{n_{i} \cup N_{i}\right\}$. 
Our FGA exploits inherent characteristics of GAs, which can efficiently explore multiple possible solutions in each evolutionary step providing a set of desired solutions at the end of its computation. The fitness function used by our FGA is based on the virtual forces envisioned to be inflicted on a mobile node by its neighbors. The virtual force $F_{i j}$ exerted on node $n_{i}$ by node $n_{j} \in$ $N_{i}$ is calculated according to the following equation

$$
F_{i j}= \begin{cases}\gamma_{i}\left(R_{c}-d_{i j}\right) & \text { if } 0<d_{i j}<d_{t h} \\ \epsilon & \text { if } d_{t h} \leq d_{i j} \leq R_{C}\end{cases}
$$

where $d_{i j}$ is the distance between mobile nodes $n_{i}$ and $n_{j}, d_{t h}$ is the threshold value to define the best separation among nodes, and $\epsilon<R_{c}-d_{t h}$. The force scaling factor $\gamma_{i}$ is a function of the desired node degree $\mu$ and is defined as

$$
\gamma_{i}=\frac{\left(\left|N_{i}\right|-\mu\right)^{2}+1}{\left|N_{i}\right|}
$$

The fitness of node $n_{i}$ located in $s_{i}$ is influenced by its neighboring node positions represented by $\boldsymbol{s}_{-i}$, where $\boldsymbol{s}_{-i}$ is an element in the set of all possible choices of $n_{i}$ 's opponents $\bar{S}_{-i}$. The fitness of $n_{i}$ is calculated as

$$
f_{i}\left(s_{i}, \boldsymbol{s}_{-i}\right)= \begin{cases}\sum_{n_{j} \in N_{i}} F_{i j} & \text { if } N_{i} \neq \varnothing \\ \mathcal{F}_{\max } & \text { otherwise }\end{cases}
$$

where $\mathcal{F}_{\max }>\left(m \times R_{C}\right)$ is a large penalty for mobile nodes becoming disconnected.

The fitness function in our FGA promotes a sparsely connected network topology with nodes having a limited number of neighbors and reduced overlapping communication areas when the desired number of neighbors $\mu$ is small. On the other hand, when the desired node degree is large, nodes running our BioGame will create a densely packed network, where each node has multiple neighbors, hence redundant routing paths can be established.

Figure 3 shows the surface plot of the fitness function defined by Eq. (8) for various degrees of node $n_{i} \in I$ and averaged distances to its neighbors in range $\left(0, R_{c}\right]$.

We can observe in Figure 3 that the fitness for node $n_{i}$ improves when its number of neighbors approaches $\mu$ and the distance to them gets closer to $R_{C}$ (i.e., nodes became spread farther apart). At any stage of the game, a player may not have the entire landscape of possible solutions to choose from, as neighbor positions may restrict it, but even a local improvement shifts the node closer to a position with the minimal virtual force inflicted on it. 


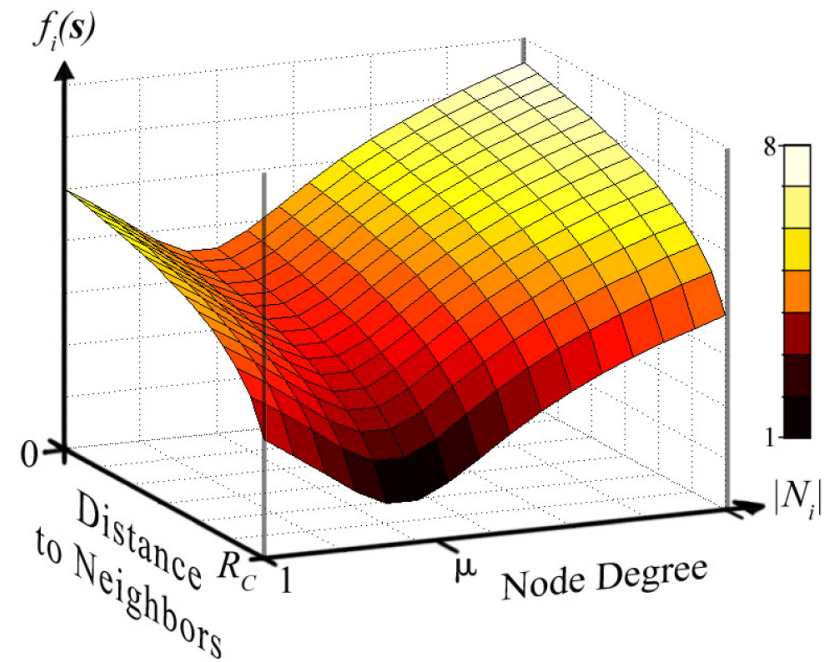

Figure 3 Fitness landscape of our FGA. For clarity of presentation, this figure does not depict the case where $N_{i}=\emptyset$, which results in $f_{i}(\boldsymbol{s})=\mathcal{F}_{\max }$.

The set of possible new locations $\bar{S}_{i}$ for node $n_{i}$ consists of locations computed by our FGA as well as $n_{i}$ 's current position. Node $n_{i}$ computes expected payoff for each $s_{i} \in \bar{S}_{i}$ as

$$
u_{i}\left(s_{i}, \sigma_{-i}\right)=\sum_{s_{-i} \in \bar{S}_{-i}}\left(\prod_{n_{j} \in N_{i}} \sigma_{j}\left(s_{j}\right)\right) f_{i}\left(s_{i}, \boldsymbol{s}_{-i}\right)
$$

where $\boldsymbol{\sigma}_{-i}$ is a probability distribution over $\boldsymbol{s}_{-i}$ and a probability of node $n_{j}$ choosing location $s_{j}$ is denoted by $\sigma_{j}\left(s_{j}\right)$, which represents a probability of $s_{j}$ being played.

Player $n_{i}$ finds the best location to move $s_{i}^{*}$ by evaluating all elements of $\bar{S}_{i}$ using Eq. (9) and selecting

$$
s_{i}^{*} \in \underset{s_{i} \in \bar{S}_{i}}{\operatorname{argmin}} u_{i}\left(s_{i}, \sigma_{-i}\right)
$$

that minimizes possible forces inflicted on it.

This step replaces the stochastic roulette wheel or deterministic elitism selection mechanisms in making a final decision for the new position of node $n_{i}$. However, contrary to the roulette wheel and elitism, our BioGame utilizes additional information about neighbors in order to enhance FGA performance. 
In our BioGame, each node $n_{i}$ autonomously determines its new location that is within $R_{C}$ distance from its current coordinates.

\subsection{Formal Analysis of BioGame}

Let us now demonstrate that BioGame can be used by autonomous mobile nodes to efficiently disperse over an area of deployment while achieving uniform distribution and maintaining network connectivity. Let $f_{i}\left(s^{t}\right)$ represent the fitness of node $n_{i}$ resulted from the strategy $s_{i}^{t}$ being played by it at time $(t)$. The following theorem shows that a mobile node moves to a new location only if it does not lower its fitness.

Theorem 1. Node $n_{i}$ moves to a new location $s_{i}^{t+1}$ if it is expected to be better or at least as good as its present position $s_{i}^{t}\left(i . e ., f_{i}\left(\boldsymbol{s}^{t+1}\right) \leq f_{i}\left(\boldsymbol{s}^{t}\right)\right)$.

Proof. Node $n_{i}$ computes expected payoffs (fitness) for all of its possible next locations $\bar{S}_{i}$ according to Eq. (9). From the expected payoffs, node $n_{i}$ selects the best location to move, as presented in Eq. (10). If there is no element in $\bar{S}_{i}$ for which Eq. (9) attains a smaller (i.e., better) value than for $s_{i}^{t}$, then $s_{i}^{t}=s_{i}^{*}$ or $u_{i}\left(s_{i}^{t}, \sigma_{-i}\right)=u_{i}\left(s_{i}^{*}, \sigma_{-i}\right)$. Hence, either node $n_{i}$ remains in its current position or moves to a location that gives it equally good or better expected payoff.

If, on the other hand, $s_{i}^{t}$ is not amongst the locations that provide the minimum expected payoff for node $n_{i}$, the new location $s_{i}^{*}$ selected by Eq. (10) must result in a better or equal expected payoff for $n_{i}$ than $s_{i}^{t}$ (i.e., $\left.u_{i}\left(s_{i}^{t}, \boldsymbol{\sigma}_{-i}\right)>u_{i}\left(s_{i}^{*}, \boldsymbol{\sigma}_{-i}\right)\right)$.

Therefore, node $n_{i}$ moves to a new location if and only if it has at least as good fitness as its current position.

We formalize the advantages of BioGame over FGA in the following theorem.

Theorem 2. In BioGame, the decision to determine the next position for player $n_{i}$ provides similar or better results than a position that is based on the outcomes of FGA only.

Proof (sketch). Let us first assume that player $n_{i}$ is the only node intending to change its location for a given moment and, consequently, no information about eventual actions of the players in $N_{i}$ provide additional information for $n_{i}$. Since $\bar{S}_{-i}$ is a singleton and $\forall_{n_{j} \in N_{i}} \sigma_{j}\left(s_{j}^{t}\right)=1$, where $s_{j}^{t}$ represents the present location of player $n_{j}$, Eq. (9) becomes equivalent to Eq. (8) and, 
hence, player $n_{i}$ selects the best new place for her to move, as ensured by Eq. (10), as if it were by using the results of our FGA only.

If, on the other hand, there is at least one other player $n_{j} \in N_{i}$ at this time intending to move according to her strategy $\sigma_{j}$, it is possible that this information can improve $u_{i}$ selection process by using BioGame. Let $\hat{s}_{i}$ be the best strategy that FGA can find (either, as an outcome of elitism, roulette wheel, or similar processes), then the expected payoff resulting from moving into $\hat{s}_{i}$ evaluated by Eq. (9) can be at most as good as the result of Eq. (10) evaluated by our BioGame. Therefore, $u_{i}\left(\hat{s}_{i}, \sigma_{-i}\right) \geq u_{i}\left(s_{i}^{*}, \sigma_{-i}\right)$ must hold.

As a result, player $n_{i}$ can find the next best location to move by evaluating her future positions with respect to possible movements of all $n_{j} \in N_{i}$ through evaluating our BioGame.

The following theorem illustrates that the mobile nodes running BioGame make better informed movement decisions than the nodes that make their choices regarding next positions based on FGA only.

Theorem 3. For any given two neighboring nodes $u_{i}$ and $u_{j}$, both at nonideal locations, BioGame provides better informed movement decisions than FGA.

Proof (sketch). Using Eq. (8), each node running FGA computes its next position regardless of possible actions of its neighbors. Therefore, it is possible that two nodes $u_{i}$ and $u_{j}$ may move to new locations which improve their own fitness but decrease the fitness and uniformity of the entire network. This can happen because each node only selfishly considers the improvement of its own location in its fitness calculation. Consider two nodes $u_{i}$ and $u_{j}$, that are attempting to move and guided by FGA. It is possible that they may choose the same location as their target $s^{t+1}$ (i.e., $s^{t+1}=s_{i}^{t+1}=s_{j}^{t+1}$ ) if the location $s^{t+1}$ provides improvement for both nodes over their respective current positions $s_{i}^{t}$ and $s_{j}^{t}$. The nodes $u_{i}$ and $u_{j}$ will then have to fix their positions at time $(t+2)$, as FGA will guide them to better successive positions.

However, a node running BioGame takes into account the intended decisions of neighboring nodes using Eq. (9). As long as the expected payoff is better for one node to move to a given location than the expected payoff of a node and its neighbors moving into the same location, nodes will not move into the same location. Therefore, as long as $\sigma_{j}\left(s^{t+1}\right) f_{i}\left(s_{i}^{t+1}, s_{j}^{t+1}\right)>$ $\sigma_{j}\left(s^{t+1}\right) f_{i}\left(\hat{s}_{i}^{t+1}, s_{j}^{t+1}\right)$, node $u_{i}$ will refrain from moving to $s_{i}^{t+1}$ in favor of moving to $\hat{s}_{i}^{t+1}$, as assured by Eq. (10). 
As a direct result of Theorem 3, we can state the following corollary regarding the performance of our BioGame.

Corollary 1. BioGame achieves convergence faster than FGA for autonomous mobile nodes spreading themselves to a desired network configuration.

Theorems 1, 2, 3, and Corollary 1 state that player $u_{i}$ can improve its performance by executing BioGame to determine the best next position to move and improve the network convergence time. This observation has been further validated by the results of our simulation experiments presented in Sect. 5 below.

\section{Simulation Experiments}

We developed a simulation platform for our BioGame using MASON [20]. Our software implementation provides a graphical user interface allowing for a real-time visualization of ongoing network dynamics and collecting data needed for further analysis. All of our experiments were performed for MANETs with autonomous nodes determining their next locations by means of BioGame and FGA alone. To reduce noise in the collected data, each experiment was performed 20 times and the results were averaged.

For each experiment, we initially placed 40 nodes in the upper-left corner of the $100 \times 100$ units deployment area (Figure 4(a)). For simplicity and without loss of generality, all mobile agents have the same communication radius of $R_{C}=16$. Our initial distribution imitates a realistic situation where the nodes enter a terrain from a common point (e.g., initiating nodes into a post-earthquake zone or a territory occupied by hostile forces) compared to random or other initial node deployments we often see in the literature. Deployed autonomous mobile nodes have no a priori knowledge of the underlining area and locations of their neighbors. A typical final distribution of 40 nodes running BioGame for 100 steps is shown in Figure 4(b).

\subsection{NAC Improvement for Networks Running BioGame and FGA}

Figure 5 shows the improvement of NAC for networks where nodes are running BioGame and FGA. In Figure 5, the vertical axis represents the ratio of the total deployment terrain covered by nodes and the horizontal axis represents the duration of the experiments. We can see in Figure 5 that mobile nodes directed by our BioGame converge faster than when mobile nodes that are directed by FGA. Also, it can be observed in Figure 5 that in the early 


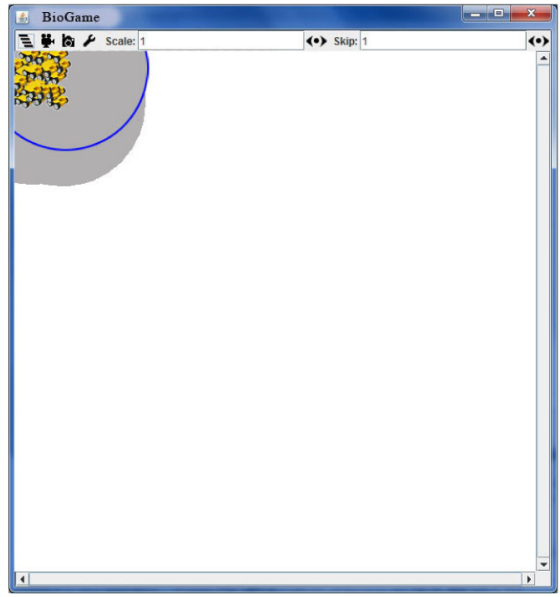

(a)

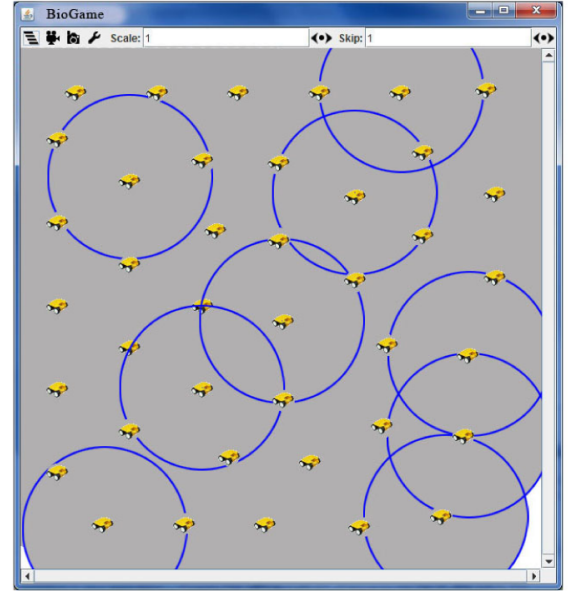

(b)

Figure 4 A typical (a) initial and (b) final node distribution of 40 nodes and their communication areas (darker color) at the beginning and end of the BioGame experiments. To better visualize BioGame performance, only the communication areas of a few selected nodes are outlined.

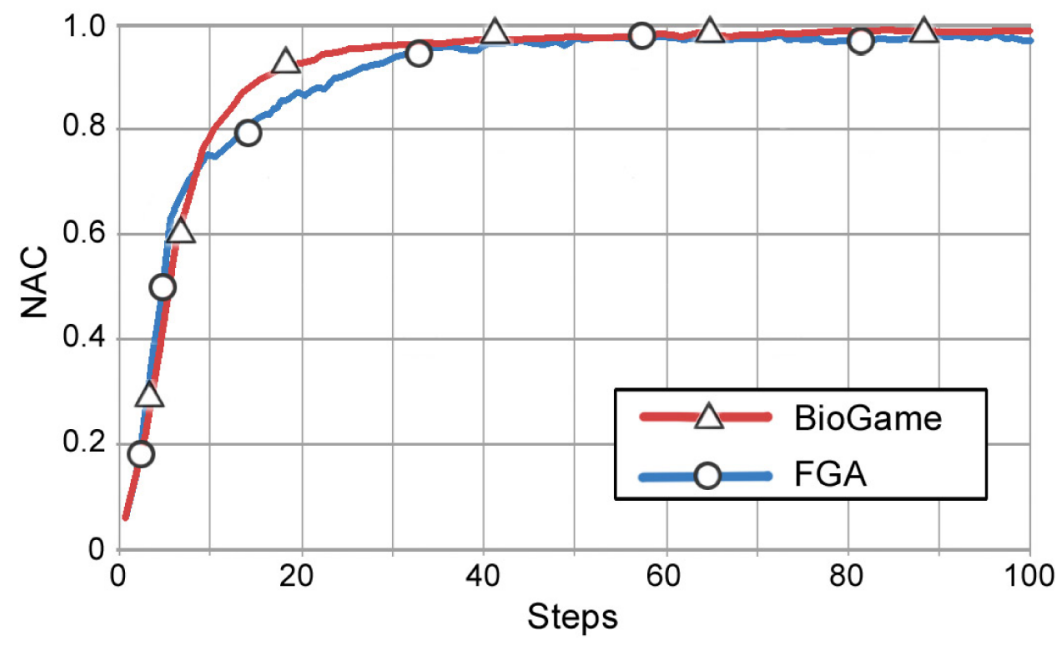

Figure 5 NACs obtained by networks running BioGame and FGA. 


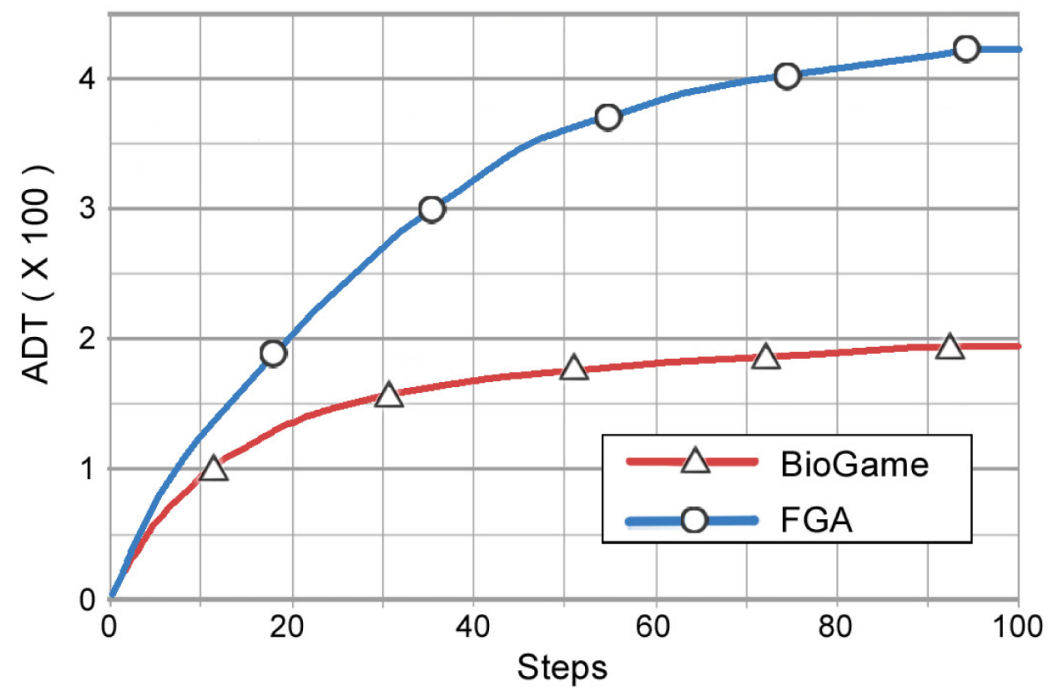

Figure 6 ADTs for a node running BioGame and FGA.

stages of the experiments, the NAC for BioGame and FGA have the highest improvement rate, indicating that the nodes are able to disperse far from their initial locations especially at the beginning of the experiments and showing effectiveness of both BioGame and FGA in finding new positions.

\subsection{Average Distance Traveled by each Node}

When illustrating changes in ADT for our experiments, the vertical axis represents the average total distance traveled by a node up to the time indicated in the horizontal axis. Figure 6 compares ADT for nodes running BioGame and FGA in a network consisting of 40 mobile agents. As we could observe in Figure 5, the area covered by mobile agents running FGA and BioGame are very similar. However, Figure 6 shows that the average distance traveled by a node running FGA is almost twice of that for BioGame. Specifically, Figure 6 shows that at step 35, ADT by a node running FGA is approximately 300 whereas it is about 160 for a node running BioGame. At step 50, when FGA and BioGame networks approach their maximum area coverages for this example (Figure 5), a node selecting its next location based on FGA traveled more than twice the distance of a node using BioGame (Figure 6). Conversely, by the time BioGame achieves $98 \%$ of coverage by traveling 
distance of approximately 160, FGA has only achieved $78 \%$ of area coverage (i.e., Figure 6, shows that FGA network ADT is 160 at step 15). The ability of BioGame to significantly reduce the required distance that nodes have to travel to accomplish predefined coverage objectives assures its practical value for all realistic implementations for which power is a scarce resource.

Another observation that we can make from Figure 6 is that ADT continues to increase throughout the experiment when mobile nodes use FGA to guide their movements. This observation shows that the nodes running FGA need more time to attain a uniform network topology than the BioGame nodes. One reason for the lower performance of FGA is that multiple nodes simultaneously may attempt to move to the same location, and delay uniform node distribution. These types of inefficient movements are greatly reduced by BioGame, since each node considers its own decisions and future actions of its near neighbors.

\subsection{BioGame and FGA Uniformity Evaluation}

We demonstrate the improvement in network uniformity when mobile nodes utilize BioGame and FGA to evolve towards their final distributions by using the metrics $\mathcal{U}_{A}$ and $\mathcal{U}_{C}$, which were presented in Sect. 3.1. Figure 7 shows the improvement of $\mathcal{U}_{A}$ and $\mathcal{U}_{C}$ as simulation experiments progress. We can see in Figure 7 that both BioGame and FGA converge rapidly towards a uniform distribution over the area of deployment. The largest improvement occurs during the initial iterations of the simulations showing the effectiveness of our approaches in finding new locations when the space is not constrained. However, as ADT results demonstrated, BioGame provides a more efficient method for spreading autonomous mobile agents over an unknown terrain since the nodes utilizing BioGame move less while providing better results with respect to NAC and appropriate separation among the mobile nodes (Figure 7).

\section{Concluding Remarks}

We presented quantitative techniques for gauging the performance of MANET nodes with respect to the uniform distribution of mobile assets, total terrain covered by communication areas of all nodes (NAC), and the distance that each node travels before a desired network topology is reached (ADT). A uniform distribution of mobile agents prolongs network's lifespan by ensuring even depletion of energy resources available to each node. We demonstrated 


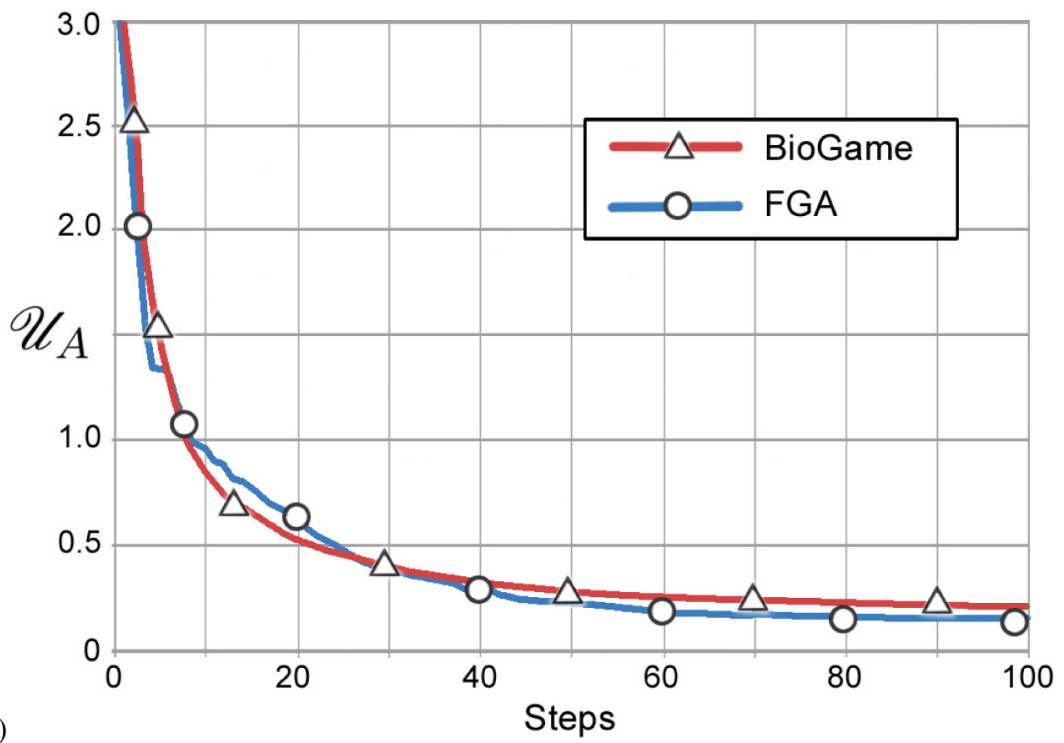

(a)

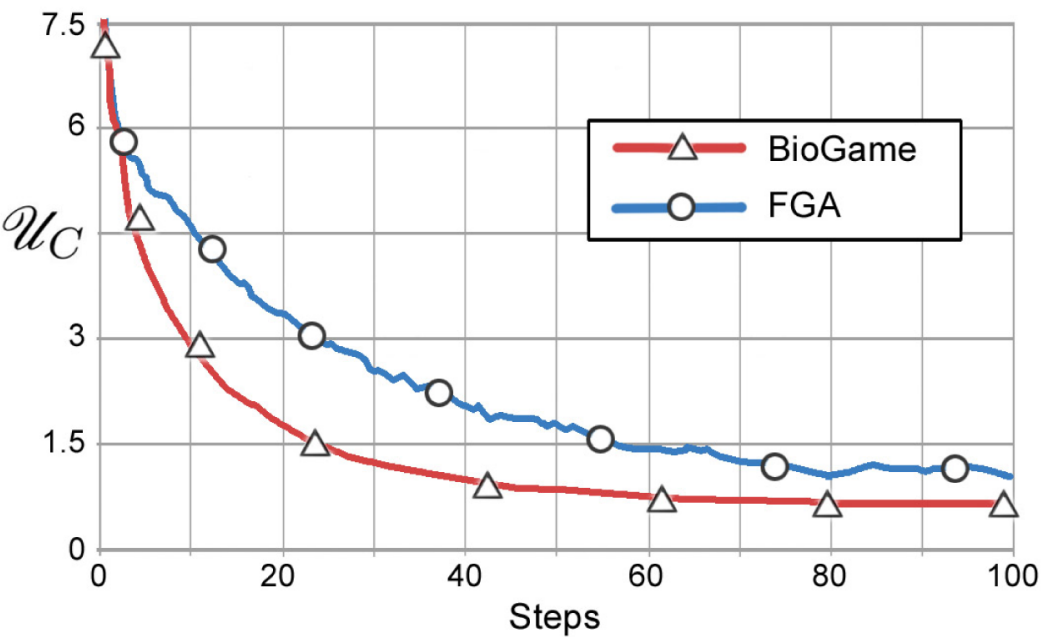

(b)

Figure 7 The improvement of uniformities (a) $\mathcal{U}_{A}$ and (b) $\mathcal{U}_{C}$ for MANETs running BioGame and FGA. 
uniformity metrics that exploit Voronoi tessellations of a deployment territory to evaluate regularity in the placement of nodes. ADT can be used to measure power-efficiency of a node distribution, as movement of the nodes is one of the most power-consuming tasks. In order to adequately utilize existing mobile agents, an autonomous node self-positioning method should strive to maximize the total area covered by all nodes while preserving network connectivity. We define NAC metric as a ratio of area covered by all nodes with respect to the deployment territory. Each performance metric gives a valuable insight into the mobile network performance and collective examination of their respective results provides a comprehensive assessment of MANETs.

We present a node-spreading bio-inspired game (BioGame) combining our force-based genetic algorithm (FGA) and game theory to guide autonomous mobile agents in modeling movement decisions. Using our MANET evaluation metrics, we compare the performance of BioGame and FGA. Experimental results show that both BioGame and FGA can provide promising levels of area coverage with near uniform node distributions. However, BioGame can achieve a network topology where nodes uniformly cover the deployment terrain while traveling less than half of the distance than mobile nodes running FGA to achieve similar uniformity and NAC results. Furthermore, simulation results demonstrate that the presented metrics are useful for evaluating the performance autonomous mobile node deployment mechanisms.

\section{Acknowledgements}

Earlier versions of this work was supported by U.S. Army CommunicationsElectronics RD\&E Center contracts W15P7T-09-CS021 and W15P7T-06C-P217, and by the National Science Foundation grants ECS-0421159 and CNS-0619577. The contents of this document represent the views of the authors and are not necessarily the official views of, or are endorsed by, the U.S. Government, Department of Defense, Department of the Army or the U.S. Army Communications-Electronics RD\&E Center.

\section{References}

[1] C. Abbas, M. Dzulkifli, and A. Azizah. Exploiting Voronoi diagram properties in face segmentation and feature extraction. Pattern Recognition, 41(12):3842-3859, 2008. 
[2] B. A. Bash and P. J. Desnoyers. Exact distributed Voronoi cell computation in sensor networks. In Proceedings of the 6th International Conference on Information Processing in Sensor Networks (IPSN'07), pages 236-243. ACM, New York, 2007.

[3] J. Bernauer, R. P. Bahadur, F. Rodier, J. Janin, and A. Poupon. DiMoVo: a Voronoi tessellation-based method for discriminating crystallographic and biological proteinprotein interactions. Bioinformatics, 24(5):652-658, March 2008.

[4] J. B. D. Cabrera, R. Ramanathan, C. Gutierrez, and R. K. Mehra. Stable topology control for mobile ad-hoc networks. Communications Letters, IEEE, 11(7):574 -576, july 2007.

[5] U. K. Chakraborty, S. K. Das, and U. T. E. Abbott. Clustering in mobile ad hoc networks with differential evolution. In Proceedings 2011 IEEE Congress on Evolutionary Computation (CEC), pages 2223-2228, June 2011.

[6] J. Cortés, S. Martinez, T. Karatas, and F. Bullo. Coverage control for mobile sensing networks. IEEE Transactions on Robotics and Automation, 20(2):243-255, April 2004.

[7] Q. Du, V. Faber, and M. Gunzburger. Centroidal voronoi tessellations: Applications and algorithms. Society for Industrial and Applied Mathematics Review, 41(4):637-676, 1999.

[8] D. Fudenberg and J. Tirole. Game Theory. The MIT Press, August 1991.

[9] B. A. Garro, H. Sossa, and R. A. Vazquez. Path planning optimization using bioinspirited algorithms. In MICAI'06: Proceedings of the Fifth Mexican International Conference on Artificial Intelligence, pages 319-330. IEEE Computer Society, Washington, DC, USA, 2006.

[10] W. George and Barlow. Hexagonal territories. Animal Behaviour, 22, Part 4:876-IN1, 1974.

[11] S. Gundry, J. Kusyk, J. Zou, C. S. Sahin, and M. U. Uyar. Performance evaluation of differential evolution based topology control method for autonomous MANET nodes. In Proceedings IEEE Symposium on Computers and Communications (ISCC), pages 228-233, May 2012.

[12] S. Gundry, J. Zou, J. Kusyk, J. Zou, C. S. Sahin, and M. U. Uyar. Markov chain model for differential evolution based topology control in MANETs. In Proceedings IEEE Sarnoff Symposium, pages 1-5, May 2012.

[13] J. H. Holland. Adaptation in Natural and Artificial Systems: An Introductory Analysis with Applications to Biology, Control and Artificial Intelligence. MIT Press, Cambridge, MA, April 1992.

[14] A. Howard, M. J. Mataric, and G. S. Sukhatme. Mobile sensor network deployment using potential fields: A distributed, scalable solution to the area coverage problem. In Proceedings Distributed Autonomous Robot Systems 5, pages 299-308, 2002.

[15] J. Kusyk, C. S. Sahin, M. U. Uyar, E. Urrea, and S. Gundry. Self organization of nodes in mobile ad hoc networks using evolutionary games and genetic algorithms. Journal of Advanced Research. Elsevier, 2:253-264, July 2011.

[16] J. Kusyk, C. S. Sahin, J. Zou, S. Gundry, E. Urrea, and M. U. Uyar. Game theoretic and bio-inspired optimization approach for autonomous movement of MANET nodes. In Handbook of Optimization, from Classical to Modern Approach, Intelligent Systems Reference Library, Vol. 38, Springer, 2011.

[17] J. Kusyk, E. Urrea, C. S. Sahin, and M. U. Uyar. Game theory and genetic algorithm based approach for self positioning of autonomous nodes. International Journal of Ad Hoc \& Sensor Wireless Networks. Old City Publishing, 16(1-3):93-118, 2012. 
[18] J. Kusyk, J. Zou, S. Gundry, C. S. Sahin, and M. U. Umit. Techniques for performance evaluation of self-positioning autonomous MANET nodes. In Proceedings IEEE Sarnoff Symposium, pages 1-5, May 2012.

[19] L. Lu, B. Lévy, and W. Wang. Centroidal Voronoi tessellations for line segments and graphs. In Computer Graphics Forum (Eurographics Conf. Proc.), 2012.

[20] S. Luke, C. Cioffi-Revilla, L. Panait, K. Sullivan, and G. Balan. MASON: A multiagent simulation environment. Simulation, 81(7):517-527, 2005.

[21] M. Mitchell. An Introduction to Genetic Algorithms. MIT Press, Cambridge, MA, 1998.

[22] H. Nguyen, J. Burkardt, M. Gunzburger, L. Ju, and Y. Saka. Constrained CVT meshes and a comparison of triangular mesh generators. Computetional Geometry, 42(1):1-19, January 2009.

[23] A. Okabe, B. Boots, and Ks Sugihara. Spatial Tesselations: Concepts and Applications of Voronoi Diagrams, Wiley Series in Probability and Mathematical Statistics. Wiley, 1992.

[24] C. S. Sahin. Genetic Algorithms for Topology Control Problems. LAP LAMBERT Academic Publishing, February 2011.

[25] C. S. Sahin, E. Urrea, M. U. Uyar, M. Conner, I. Hokelek, G. Bertoli, and C. Pizzo. Genetic algorithms for self-spreading nodes in MANETs. In Proceedings of the 10th Annual Conference on Genetic and Evolutionary Computation (GECCO), pages 1141$1142,2008$.

[26] J. Toutouh and E. Alba. Optimizing OLSR in VANETS with differential evolution: a comprehensive study. In Proceedings of the First ACM International Symposium on Design and Analysis of Intelligent Vehicular Networks and Applications (DIVANet'11), pages 1-8. ACM, New York, 2011.

[27] E. Urrea, C. S. Sahin, I. Hokelek, M. U. Uyar, M. Conner, G. Bertoli, and C. Pizzo. Bio-inspired topology control for knowledge sharing mobile agents. Ad Hoc Networks, 7(4):677-689, 2009

[28] A. R. Vahdat, A. N. NourAshrafoddin, and S. S. Ghidary. Mobile robot global localization using differential evolution and particle swarm optimization. In Proceedings IEEE Congress on Evolutionary Computation (CEC2007), pages 1527-1534, Sept. 2007.

[29] Y. Wang, L. Cao, and T. A. Dahlberg. Efficient fault tolerant topology control for threedimensional wireless networks. In Proceedings of 17th International Conference on Computer Communications and Networks (ICCCN '08), pages 1-6, Aug. 2008.

[30] J. Zou, S. Gundry, J. Kusyk, M. U. Uyar, and C. S. Sahin. 3D genetic algorithms for underwater sensor networks. International Journal of Ad Hoc and Ubiquitous Computing, 13(1):10-22, 2013.

\section{Biographies}

Janusz Kusyk, Ph.D., received B.S. and M.A. degrees in Computer Science from Brooklyn College, Brooklyn, New York in 2002 and 2006, respectively, and he received Ph.D. degree in Computer Science in the Graduate Center, The City University of New York in 2012. Currently, he is a Patent Examiner at USPTO, Alexandria, VA. His research interests are in the areas of network 
modeling and analysis and applications of game theory and genetically inspired algorithms to wireless networks and distributed robotics.

Jianmin Zou received his B.S. degrees in both Computer Science and Chemical Engineering from Huazhong University of Science and Technology, P. R. of China in 2009. He is currently a Ph.D. candidate at the City College of New York (CCNY) of the City University of New York (CUNY). His interests include wireless mobile ad hoc networks, underwater sensor networks, biologically inspired algorithms and game theory.

Stephen Gundry received two Bachelor of Science degrees in both Engineering Science and Physics from the City University of New York at the College of Staten Island (CSI), in 2003, and a Master of Engineering degree in Electrical Engineering from the City University of New York at the City College of New York (CCNY), in 2009 and is currently a Ph.D. candidate at this institution. His interests include biologically inspired algorithms, artificial intelligence, game theory and mobile ad hoc networks.

Cem Safak Sahin, Ph.D., received his B.S. degree from Gazi University, Turkey in 1996, M.S. degree from Middle East Technical University, Turkey in 2000, and M. Phil. and Ph.D. degrees from the City University of New York in 2010, all in Electrical Engineering. Until 2004 he was an engineer at Roketsan Inc., a leading defense company of Turkey's rocket and missile research and production programs. From 2004 to 2008, he was Principal Engineer, Systems Design at Mikes Inc., a defense company specializing in Electronic Warfare Systems, working as part of a multi-national defense project in the United States. He was Senior Software Engineer from 2008 to 2010 in Elanti System. Currently, he is Senior Research Engineer at BAE Systems-AIT in Burlington, MA. His interests include wireless ad-hoc networks, bio-inspired algorithms, communication theory, multi-sensor fusion, algorithm development, artificial intelligence, machine learning, and electronic warfare systems.

M. Ümit Uyar, Ph.D., is a Professor with the Electrical Engineering Department of the City College and the Computer Science Department of the Graduate Center of the City University of New York. His interests include bio-inspired computation with applications to the mobile ad hoc networks, distributed robotics tasks and cancer chemotherapy treatment decision support systems. Dr. Uyar was the lead principle investigator for several large 
grants from U.S. Army and NSF to conduct research on knowledge sharing mobile agents using bio-inspired algorithms for topology control in MANETs and for a smart robot brain on FPGA which has reliable communication capabilities. Prior to joining academia, he was a Distinguished Member of Technical Staff at AT\&T Bell Labs until 1993. He is an IEEE Fellow and holds six U.S. patents. Dr. M. Umit Uyar has a B.S. degree from Istanbul Technical University, and M.S. and Ph.D. degrees from Cornell University, Ithaca, NY, all in electrical engineering. 